\title{
Saisir la vie amoureuse par le croisement des méthodes
}

\author{
Anne-Cécile Mouget ${ }^{1}$
}

\begin{abstract}
[Résumé] Dans cet article, je souhaiterais montrer l'intérêt de croiser plusieurs méthodes de recueil de données pour étudier un processus interpersonnel comme l'amour. De l'amour, il n'est question ici que de l'amour eros, l'amour entre partenaires, ce que les anglophones appellent romantic relationships. La réflexion se limite aussi uniquement à des sociétés occidentales, mes terrains comprenant la France, les USA (principalement la Californie), et l'Espagne (principalement la Catalogne). L'expérience amoureuse est à la fois répandue, partagée, ordinaire, elle est co-construite dans des interactions, mais elle est aussi personnelle, intime et profonde. La Grounded Theory offre un cadre méthodologique permettant de gérer la variété des représentations de l'amour. Mais elle ne présume pas des techniques de recueil de données à mettre en œuvre. Au contraire, elle laisse la possibilité de travailler sur des données diversifiées afin d'explorer les différentes dimensions de l'amour. J'exposerai ici mon propre cheminement méthodologique, suivant l'ordre chronologique du déploiement de la recherche que je mène dans le cadre d'un doctorat en sociologie sur la vie amoureuse d'hommes lésés médullaires. II s'est trouvé que cet ordre suive aussi une logique dans le type de données recueillies: public, privé, intime, des données dont les apports complémentaires permettent de pleinement saisir les histoires de vie amoureuse.

Mots-clés : amour, autoethnographie, grounded theory, handicap, méthodologie.
\end{abstract}

[Abstract] In this article, I would like to explain how the crossing of different data gathering methods can improve the study of romantic relationships. I will only talk about Western societies, my fieldwork taking place in France, USA (mainly California) and Spain (mainly Catalonia). Love is an inter-personal process commonly experienced, but it is also very personal and intimate. Grounded Theory offers a methodological frame for dealing with the variety of representations of love. But it does not suggest which are the best techniques for data gathering. On the contrary, it leaves the possibility of working on diversified data in order to explore the various dimensions of love. I shall expose here my own methodological path, following the chronological order of the study that I conduct for a PhD in Sociology on the subject of the love life of $\mathrm{SCl}$ men. It turned out that this order is also logical, going from public through private data, until intimate ones. They all complement each other disclosing how love life stories take place.

Keywords: love, autoethnography, Grounded Theory, disabilities, methodology. 


\section{Histoire du projet}

Ma vie a profondément changé lorsque je suis devenue handicapée. J’ai perdu une grande part de ce que j'étais, de ce que j'avais ou que j'aimais. À l'aube de ma «vie 2.0 ", comme disent mes informateurs, j'ai eu une relation amoureuse avec un homme paraplégique. À partir de nos échanges, j’ai commencé un roman dont le héros est lui aussi paraplégique. Je souhaitais que mes descriptions soient fidèles à ce qu'un homme paraplégique pouvait effectivement vivre. Aujourd'hui, je dirais des descriptions qu'elles sont vérisimilaires. Même sans être réel, un roman peut présenter des situations vraies. Pour améliorer cette vérité, j’ai cherché d'autres histoires que la nôtre. Un nouveau réseau de proches s'est construit autour de moi et de mon travail qui a pris peu à peu une dimension professionnelle et militante. Je me suis remise à lire des ouvrages théoriques et, une fois mon manuscrit achevé, j’ai compris qu'il s'agissait d'un roman ethnographique. Mes amis m'ont incitée à m'inscrire en doctorat sur le sujet de la vie amoureuse et sexuelle des hommes lésés médullaires (paraplégiques, tétraplégiques, spina bifida et d'autres causes médicales plus rares). J'ai ainsi commencé ma thèse avec des terrains, des informateurs et des données documentaires: livres, films, vidéos YouTube, messages de forums, billets de blog, etc. J'avais aussi les souvenirs des histoires réunies pour écrire mon roman.

J'entrevoyais déjà quelques difficultés à avoir choisi l'amour comme objet de recherche. Dans nos sociétés occidentales, l'amour romantique est censé présider aux unions et justifie le «bon » sexe. Que l'amour soit ainsi valorisé en fait une expérience répandue qui nous touche tous, même indirectement. Toute notre vie nous vivons avec (ou sans) amour, entourés des couples formés par les autres. L'amour est un état qui se poursuit même lorsqu'on se livre à d'autres activités. Il occupe donc une grande part du quotidien. L'expérience est longue et continue, si ordinaire que sa forme en semble naturelle. Nous partageons notre expérience amoureuse, elle est un sujet de conversation et d'expression, elle fait produire des objets culturels, artistiques, commerciaux, publics ou personnels, servant dans des rites et des fêtes. On y consacre de l'argent, du temps, de l'énergie et parfois sa vie entière. Dans cette perspective les Espagnols appellent l'être aimé « mi vida » et lui déclarent : « me muero por ti ». Comme le chante John Paul Young: "Love is in the air » ou Francis Cabrel : "L'amour est partout où tu regardes ». Sa présence généralisée elle-même est culturelle. En poursuivant ma recherche, différentes questions sont apparues. Comment gérer la multiplicité de ces représentations ? Comment pénétrer les différents niveaux personnels, culturels, et surtout, celui qui m’intéresse le plus, le niveau interpersonnel de la relation, de l'interaction amoureuse, où viennent pleinement interférer les contraintes imposées par le handicap ? Comment accéder à ce qui relève du personnel, du privé, de l'intime dans l'amour? Comment accéder aux pratiques dont certaines sont, par essence, inobservables (les relations intimes...) ? Que faire, en tant que chercheur, de sa propre expérience de l'amour? Mes réponses à ces questions ont consisté en une succession de choix méthodologiques. 
À ce foisonnement de questions, venait se mêler le constat de l'impact des particularités du groupe étudié sur le recueil de données. Dès le départ j'avais choisi de limiter ma recherche à des personnes s'identifiant comme «hommes », j'avais l'intuition que l'étude du nœud masculinité/dominant handicap/minorisé serait fructueuse. L'étude des statistiques existantes a confirmé la pertinence de ce choix. En effet le retentissement négatif du handicap moteur associé à la dépendance matérielle et/ou humaine sur la vie de couple est plus sensible pour les hommes. Bien qu'ils aient souvent une vie amoureuse (l'amour n'a pas besoin d'être réciproque) les hommes handicapés moteurs ont un accès limité à la sexualité, encore plus s'ils vivent en institution. Il leur est plus difficile de trouver de nouvelles partenaires, avec lesquelles ils forment des couples qui durent généralement moins longtemps. Ceux qui n'étaient pas entrés dans la vie sexuelle avant la survenue de leur handicap restent vierges plus longtemps, parfois pour la vie. La plupart expérimentent de longues périodes d'abstinence. Les données auxquelles accéder ne sont pas aussi abondantes que pour la population ordinaire, mais, dans de possibles « déserts affectifs » (un terme qui revient chez les informateurs), chaque relation vécue prend un relief particulier.

\section{Ancrer la théorie}

Mon premier choix méthodologique a été la grounded theory (Glaser, Strauss, 1967). En effet je souhaitais passer outre le besoin d'une « rupture épistémologique » à la Bachelard (1967). Tout d'abord, je doutais de l'efficacité de cette technique appliquée au cœur de ma vie émotionnelle. La rupture, toute relative, risquait de rester superficielle, rendant l'influence de représentations infra-conscientes, incorporées dans mes pratiques, encore plus insidieuse. Ensuite, je ne considère pas mes informateurs comme des idiots culturels, comme diraient les tenants de l'ethnométhodologie, je dois le même respect à mes propres connaissances. La Grounded Theory propose de partir sans hypothèse a priori et de s'appuyer uniquement sur des données de terrain pour construire de la théorie. Mon adhésion à une vision des savoirs humains en continuité plutôt qu'en discontinuité relève de ce que l'épistémologue Gerald Holton (1981) appelle des themata. Des croyances, allant souvent par paires opposées (comme ici continuité versus discontinuité), fondatrices de la recherche à l'échelle personnelle, ou à l'échelle de courants de pensées, dont on ne peut pas prouver la validité au détriment de l'opposé.

La grounded theory est une méthodologie d'analyse de données qui ne détermine pas quelle méthodologie de recueil doit lui être associée. La Grounded Theory structure la recherche en spirales, dans des cycles qui alternent recueil, analyse comparative et lectures théoriques. Les résultats d'un cycle d'analyse indiquent une direction pour poursuivre le recueil sur le terrain. En général cela consiste en la recherche de nouveaux cas (échantillonnage théorique) pour raffiner les hypothèses qui ont émergées. Mais comme l'expliquent Glaser et Strauss : « Avec l'échantillonnage théorique, aucun type de données relatives à une catégorie ni aucune technique de recueil n’est néces- 
sairement appropriée». (2010 : 163). Cela laisse la possibilité de construire une cohérence entre l'objet de recherche et la méthodologie de recueil.

\section{Récolter des récits de vie}

Pour formaliser mon projet de thèse, j'ai dû décider d'une méthodologie de recueil. J'ai choisi les récits de vie recueillis lors d'entretiens en face-à-face. Cette technique classique est adaptée pour comprendre les évolutions au cours d'une existence, par exemple pour faire face au handicap. Elle permet de donner la parole aux personnes concernées, c'était essentiel. En effet, le handicap est considéré comme un domaine d'« experts » et de "personnes en charge » : travailleurs sociaux, psys, médecins, parents, etc. qui, grâce à leur position de domination symbolique, sont écoutés avant les personnes elles-mêmes. Au point que certains enquêteurs interrogent les soignants pour connaître les situations de couple de personnes handicapées institutionnalisées ! Une terminologie spécifique a été créée pour désigner la vie amoureuse des personnes handicapées : la "vie affective » (accolée à " et sexuelle »). Même si le mouvement de recherche participative menée par des chercheurs et des personnes handicapées est ancien dans la sphère anglophone (Shakespeare, Gillespie-Sells, Davies, 1996) il n'est pas encore aussi répandu en France. Il était fondamental d'interroger directement les hommes lésés médullaires sur le développement de leur vie amoureuse.

Les résultats de mes premiers entretiens étaient disparates. Certains hommes ont accepté l'entretien de face-à-face, d'autres ont préféré Skype, le téléphone ou le tchat. Un homme a souhaité faire l'entretien avec sa compagne. Les messages échangés autour des entretiens étaient parfois plus intéressants que l'entretien lui-même. Heureusement c'est le moment où j'ai entendu parler de l'enquête de J. Couduriès sur la vie de couple des hommes gays. L'annexe méthodologique de son livre Être en couple (gay) (2011) m'a été d'un grand réconfort. Lorsqu'on refuse de recruter les enquêtés à partir d'un de leurs lieux de socialisation commun pour éviter la couleur que cela donnerait aux données (classiquement les associations pour les gays, les centres de rééducation pour les personnes handicapées) il faut composer avec une perte d'homogénéité dans les manières de répondre. Le sujet de l'enquête et la condition physique des personnes interrogées ont, bien sûr, aussi eu un impact sur ces manières de répondre.

Conformément à la démarche de la Grounded Theory, je comparais les premières histoires recueillies pour les analyser, mais les données affluaient et étaient de plus en plus variées. Des interviewés revenaient vers moi pour me raconter les rebondissements dans leur vie amoureuse. Certains ont gardé le contact jusqu’à devenir des informateurs privilégiés et même des amis. J'utilisais principalement les réseaux sociaux pour mon recrutement, et comme mon travail devenait de plus en plus connu dans les «milieux handis » en ligne, j’ai commencé à être contactée spontanément: «C'est toi qui fait une enquête sur nous ?» ou : «'ai eu un petit ami tétraplégique, estce que ça t'intéresse que je te raconte pour ta thèse ?». Un jour, alors que j'étais assise dans le « carré handicapé » d'un train, un homme tétraplégique a été installé en face 
de moi par les contrôleurs. Il me souriait et essayait d'engager la conversation. Il avait envie de me "raconter sa vie» comme on dit. Je ne savais pas comment gérer l'échange d'un point de vue éthique : devais-je lui parler de ma recherche et risquer de l'embarrasser ? Ne rien dire et discuter simplement ? Mais que ferai-je de ce qu'il allait me dire ? Je ne pourrais pas faire, plus tard, comme si la conversation n'avait pas eu lieu... Je réfléchissais en me concentrant ostensiblement sur le paysage, mais je me sentais impolie. Après tout bavarder avec des inconnus fait partie du charme des voyages en train. J'ai oublié mes préoccupations épistémologiques, je suis entrée dans l'échange et je l'ai écouté me dérouler son histoire. Cette rencontre a été un déclencheur. Les chemins que je parcours du fait de ma recherche et de mon propre handicap me mettent souvent dans des positions d'amie ou de confidente potentielle (voir de confesseur pour ce qui concerne la sexualité). Au lieu d'essayer de contrôler mes données pour que ma méthodologie reste "pure», je me suis mise à surfer sur la vague de biographies qui m'était offerte.

J'ai dès lors décidé d'utiliser systématiquement les textes biographiques non produits pour ma recherche. Cette part de mon corpus tient aux particularités du groupe que j'étudie. En effet les vies handicapées réunissent bon nombre des critères de succès éditorial déterminés par J. Pennef (1990). Accepter de travailler sur les autobiographies c'est bénéficier du vécu du Dr M. Maury², premier médecin tétraplégique français, de l'analyse anthropologique de R. R. Murphy, de celle, psychologique, de J-L Simon, des descriptions incisives du slameur Grand Corps Malade, de la prose de romanciers comme B. de Stabenrath ou P. Segal, de la poésie de J. Bousquet, des souvenirs érotiques de Brantôme. Aurais-je pu parler à tous ces hommes ? Certains, comme l'acteur l'acteur Ch. Reeve, sont morts, d'autres, comme le ministre allemand W. Schäuble, sont difficilement accessibles. Le batteur R. Wyatt, le pilote Ph. Streiff, l'ex membre de gang de Chicago M. Juette... autant de biographies qui ont aussi pour intérêt d'avoir été produites hors de l'influence de mes questions ou de ma présence. Cette démarche m'a permis de trouver des récits biographiques de personnes appartenant à des types rares comme un prêtre tétraplégique ou des hommes lésés médullaires gays.

Les récits de vie écrits, plus travaillés et réflexifs, sont complémentaires des entretiens, plus spontanés. Certains auteurs, parce qu'ils sont écrivains, poètes ou sensibles, font des descriptions exceptionnelles de leur vie amoureuse qui aident à la compréhension du fonctionnement subtile de l'interpersonnalité. Par exemple, c'est grâce à une métaphore que j’ai pu comprendre les négociations tacites. F. Granja, un catalan tétraplégique, décrit ainsi sa relation avec l'une de ses compagnes : « Les conversations étaient fluides et naturelles et se prolongeaient pendant des heures. Il y avait aussi des silences, que nous incorporions, comme un élément supplémentaire de

2 Afin de ne pas alourdir le texte j'ai préféré me centrer sur les hommes en tant qu'auteurs, leurs livres étant faciles à trouver grâce aux moyens de recherche actuels. 
notre interaction ${ }^{3}$ » (2013: 37). L'auteur file la métaphore d'un langage qu'il crée avec sa compagne, dans une conversation entre leurs corps: «De fait, il paraissait que nous étions en train de créer ensemble un nouveau langage. [...] Ensemble nous découvrions une route inconnue avec une personne qui, en quelque sorte, s'en retrouvait familière » (idem : 38). La perception du rapport qu'ils entretiennent comme «naturel » ou « familier » valide leurs choix créatifs et contribue à leur attachement.

J'ai complété mon corpus avec les biographies librement diffusées sur Internet (blogs, vlogs, publications kindle, etc.) car elles permettent de compenser la surreprésentation des personnalités ou des supercrips préférés par les éditeurs papier. Grâce à cette technique, dès la fin de ma première année de thèse, je disposais d'un peu plus de 160 récits de vie, un tiers, sollicités ou spontanés, qui m’ont été directement confiés, un tiers d'autobiographies longues, au moins de la taille d'un livre, un tiers de biographies courtes.

\section{Rester ouvert au monde}

J'avais désormais un corpus composite, et mes hypothèses, comme la liberté que je m'étais ouverte, m'invitaient à aller plus loin dans la recherche de cohérence entre mon objet de recherche et ma méthodologie. Si notre société a prévu qu'il n'est pas très correct d'observer les autres s'aimer (voyeurisme, indiscrétion), elle a aussi prévu des contextes où l'on peut donner à voir publiquement l'amour : les fictions sentimentales, le partage de photos ou de textes sur les réseaux sociaux, les chansons... ces sources favorisent l'imagination sociologique. Elles sont aussi les véhicules des représentations partagées par une culture à un moment donné de son histoire, par un double mouvement de collection (rien n'est jamais écrit à partir de rien) et de diffusion d'histoires, d'archétypes, de scénarios, etc. C'est déjà ce type de mythes que se proposait de synthétiser Barthes dans ses Fragments d'un discours amoureux (1977). Je préfère essayer d'y retrouver des scripts au sens de Gagnon et Simon (1973), ce concept pouvant dépasser le niveau sexuel pour s'appliquer aux scripts de séduction, scripts amoureux etc. Le concept de mythe est plus utile à l'étude de la symbolique, de la communication, alors que les aspects perceptifs ou performatifs du script permettent d'analyser le faire, ce qui est particulièrement important dans le cadre du handicap où le corps handicapé vient mettre sa réalité crue dans les rouages amoureux.

La réalité influence la production de textes et d'images artistiques, culturels qui, en retour influencent la réalité. Il peut s'agir d'une simple chanson qui des années plus tard revient à la mémoire des gens lorsqu'il est question d'un "problème ». Trois personnes différentes, en apprenant le sujet de ma thèse, m’ont immédiatement parlé de la chanson de M. Tillis : Ruby, Don't Take Your Love to Town (1969) dont les paroles ont été écrites à partir d'un fait divers : un vétéran paraplégique assassine sa femme pour

3 Traductions de l'espagnol faites par mes soins. 
être sortie avec un homme valide, puis se suicide. Sortie en pleine guerre du Vietnam, cette chanson devient un tube international. Elle correspondait aux préoccupations de nombreuses personnes qui ont pu l'intégrer à leurs représentations sur ce "problème » de la vie amoureuse des hommes paraplégiques.

Les données documentaires permettent de pénétrer le monde symbolique de pratiques et de représentations des personnes que l'on étudie, afin de pouvoir le décrire, mais aussi comprendre la configuration de normes et de valeurs qui contraignent leur action. On retrouve cette démarche dans le travail de la sociologue Eva Illouz, qui comme Roland Barthes, s'affronte à une immensité de données possibles. Le fait de se centrer sur un groupe précisément défini, comme ici des hommes présentant une même atteinte physique, limite les données et permet de les exploiter de manière systématique. Il est intéressant de constater que la question de l'amour entre une femme et un homme paraplégique existe depuis le cinéma muet (The Home Maker, The Zero Hour...) et a été traitée par les plus grands réalisateurs (Von Trier, Almodovar, Cameron...), incarnée par les plus grands acteurs (Brando, Wayne...) dans nombre de cultures occidentales: Avatar, The Men, Carne Tremula, Wings of Eagle, Breaking the Waves... autant de films largement vus qui véhiculent des représentations et des scripts puissants grâces aux images animées. Ces récits visuels sont d'autant plus importants que les figures d'identifications pour les hommes handicapés, ou leurs conjointes, sont rares. Des films de retour de guerre comme The Lucky Star, Best Years of our Lifes, Coming Home ouvrent la réflexion sur les relations possibles entre les femmes et les soldats blessés. Dans Coming Home (Ashby, 1978), J. Fonda, très militante et bien renseignée (elle s'était liée d'amitié avec R. Kowic, l'auteur de Born on the $4^{\text {th }}$ of July), joue de sa persona pour donner un guide d'interaction avec les vétérans du Vietnam. Une démarche militante dont elle fait part dans sa biographie (2005). Lorsque qu'elle laisse longuement filmer son visage dans l'extase, lors du rapport sexuel entre son personnage, Sally, et celui incarné par J. Voight, Luke, vétéran paraplégique, on revoit la belle Barbarella en train de briser l'orgasmotron... J. Fonda affirme ainsi qu'on peut avoir une relation amoureuse et sexuelle épanouie avec un homme lésé médullaire. Elle montre aussi comment : ramasser sa poche d'urine sans être dégoûtée, dîner avec lui aux chandelles en pensant à enlever une des chaises pour qu'il glisse facilement son fauteuil sous la table, s'assoir sur ses genoux pour se câliner, etc.

\section{S'enrichir des récits fictionnels}

Parmi les données documentaires, quel statut donner aux récits fictionnels ? La biographie, le reportage, le biopic, qui seraient les histoires les plus vraies, ne s'opposent pas au roman ou à la comédie sentimentale qui seraient les histoires les plus fictionnelles. Mon opinion d'une continuité dans les savoirs humains s'applique aussi aux supports où ils sont inscrits. L'étude des contextes de production de ces supports est révélatrice. On apprend ainsi que R. Kovic, probablement pour renforcer l'impression de son rejet par les femmes, a omis de parler de sa compagne dans sa biographie. C'est le texte qui a servi de base tant au biopic Born on the $4^{\text {th }}$ of July d'O. Stone (1989) qu'à 
Coming Home dont l'intrigue est différente. En effet l'écriture de son scénario a pris en compte les témoignages d'autres vétérans blessés médullaires et d'handicapés civils. Finalement, parmi ces trois histoires, c'est Coming Home, malgré son statut de fiction, qui est la plus vérisimilaire.

Le roman a une place particulière dans l'accès à l'expérience amoureuse. Sa particularité est de rendre accessibles les discours intérieurs. Lady's Chatterley's Lover de D. H. Lawrence (1928) est aussi une histoire de soldat blessé, cette fois lors de la Grande Guerre. Avec les progrès de la médecine beaucoup d'hommes qui seraient morts lors de conflits précédents ont survécu pour devenir handicapés. Réédité, traduit, porté au moins une dizaine de fois à l'écran depuis 1955, ce roman a certainement contribué à l'archétype de l'invalide aigri par son handicap, forcément impuissant et stérile, devenu incapable d'amour, négligeant les besoins émotionnels et physiques de sa femme, qu'il envoie se chercher un amant! Ainsi qu'à toutes les justifications possibles pour les conjoints dans une telle situation. Mosse (1996) a montré que la Grande Guerre a forgé la masculinité moderne à travers des processus de représentations, de célébrations, de mythifications, etc. Il est très probable que par les mêmes processus elle ait changé les représentations sur les possibilités d'amour et d'union avec les hommes handicapés. Dans le recueil de biographies La vie après l'accident la conjointe d'un homme devenu paraplégique explique : «D'ailleurs, il m'avait annoncé que s'il se retrouvait un jour en fauteuil, il chercherait à me dégoûter, pour que je m'en aille, se souvient Anne. Alors il m'a mise à l'épreuve pendant plusieurs mois. Malgré son agressivité, j'ai tenu le coup » (Koch, 1994 : 106). On voit poindre l'archétype Lord Chaterley!

\section{Utiliser les traces de discours amoureux}

« Tout ce que j'ai pu écrire / Je l'ai puisé à l'encre de tes yeux » chante Cabrel. L'écriture fait partie intégrante de nos rites amoureux romantique. Elle génère des traces qui peuvent être étudiées pour accéder, indirectement, mais sans le truchement d'un " discours sur le discours », à des dimensions privées et intimes de l'amour. Régulièrement mes informateurs m'ont indiqué faire circuler des textes au sein de leurs couples. Généralement, je connais leur fonction : une jeune femme me raconte écrire des textes érotiques à l'homme paraplégique dont elle est amoureuse afin de lui signifier que leurs rapports sexuels se passeront bien. Plus rarement, j'en connais le conte$\mathrm{nu}$ : une lettre d'amour dont on me donne une copie, une petite annonce pour un site de rencontre qu'on me demande de traduire, un SMS qu'on me montre, etc. Ces textes peuvent être considérés pour leur contenu ou comme des pratiques amoureuses, en effet la lettre, le poème... sont des offrandes romantiques. Ces textes, produits en privé, sont parfois rendus publics. Une jeune femme diffuse sur les réseaux sociaux les poèmes de son mari paralysé : «Tant que mes yeux d'amour l'abreuvent / dans cette intimité où les mots se meuvent». Comme dans ces vers, ils donnent à voir des aspects de leur relation malgré le corps immobile du partenaire masculin. 
D'autres textes m'étaient quant à eux directement adressés. Les réseaux sociaux sont devenus un de mes terrains, mais pour certains hommes handicapés moteurs c'est un espace de drague. Il m'est arrivé d'avoir des contacts où dès la troisième ligne on me demande: «Tu es célib ?» alors que mon statut (en couple) est renseigné, avec des messages comme : «Tu sais je suis très coquin, j'aime trop les femmes » ou « Je suis en manque d'une femme, tu es choquée ?" assortis de "gros bisous » et d'émoticônes roses à cœurs... et cela m’a permis de comprendre qu'une partie des hommes handicapés moteurs, faute d'un parcours ordinaire, d'expérience amoureuse, ne maîtrisent pas toujours les codes de la séduction. Je ne l'avais pas perçu dans les entretiens.

\section{Recourir à ses proches : Friendship as a method}

Certains de mes amis sont devenus des informateurs, certains de mes enquêtés sont devenus des amis. Cela tient à l'histoire de ma recherche, au fait que nous partageons la même condition de personnes handicapées, aux jeux de séduction homme / femme dont je me retrouve l'objet, au fait que je ne cache pas avoir eu des compagnons handicapés et que mon compagnon actuel est handicapé. Cela tient aussi à la gravité de mon sujet. Un homme racontant l'entièreté de son accident, qu'il n'avait fait qu'évoquer pendant des années pour préserver ses proches, les récits de premières fois, de chagrins d'amour, d'épiphanies sexuelles... les échanges sont parfois très intenses et créent une proximité humaine, qui dépasse les rapports romantiques ou érotiques hommes/femmes. Cette proximité est sûrement à l'origine des liens amicaux que j’ai noués avec une partie de mes interviewés.

La relation amicale facilite en retour certains types d'échanges ou d'observations. L'amour est un sujet de discussion légitime entre amis, bien plus qu'entre inconnus. L'amitié permet le partage de l'intimité, et des observations, au moins de fragments, d'histoires amoureuses, entre des personnes dont on connaît, au moins en partie, l'histoire. J. Couduriès rapporte, toujours dans l'annexe méthodologique de son livre (2011), avoir observé un couple d'amis construire leur vie amoureuse. C'est grâce à l'amitié que l'on m'a montré des lettres, des SMS, des cadeaux échangés, des objets utilisés, qu'on m'a invitée et que j'ai pu découvrir des intérieurs, des chambres à coucher, passer du temps avec des couples etc. C'est grâce à l'amitié que des informateurs m'ont prise comme confidente, et que j'accède à des histoires que je n'aurais jamais connues en tant que sociologue! "Il n'y a qu'avec toi que je parle de ces choses-là » m’a dit un informateur au décours d'échanges sur ses paraphilies après plusieurs mois de tchat. D'autres me confient les peines qu'ils cachent derrière un masque de « bon handicapé » toujours souriant. Un masque qu'ils auraient probablement activé en entretien. La communication médiée par ordinateur, grâce à l'abri de l'écran, contribue aussi à la profondeur des échanges. Lisa L. Tillmann a formalisé l'utilisation de l'amitié comme méthode dans sa thèse sur l'amitié hétéros / gays dans un article de 2003 : «Friendship as Method». Elle remarque que c'est un bon moyen d'accéder à la profondeur des expériences: "Friendship as method can bring us to a level of understanding and depth of experience we may be unable to reach using only traditional meth- 
ods. ». Cela grâce au temps long passé ensemble qui permet de se connaître émotionnellement.

\section{Exploiter sa propre expérience : autoethnographie}

Plus ma recherche progressait plus je me retrouvais personnellement impliquée dans le recueil de données : par les amitiés qui se nouaient ou s'approfondissaient, par les tentatives de séduction, par les échos à mes propres histoires amoureuses. Les premiers manuels de Grounded Theory ne parlent pas de la position du chercheur dans les groupes ou les interactions sociales qu'il étudie. Ils n'expliquent pas comment cela influence ses possibilités d'accéder à certaines données plus qu'à d'autres, ni n'abordent l'utilisation possible de sa propre expérience comme ressource. J'avais organisé ma lecture selon un ordre chronologique et c'est aussi en cours d'enquête que je me suis rendue compte des difficultés que posait le fait d'avoir oublié que j'existais dans le processus de recherche! Ce qui me touchait le plus était de ne pas pouvoir m'empêcher de penser à ma propre expérience : je connais les deux positions d'aimer une personne handicapée et d'être aimée en tant que personne handicapée. Je revivais ce que Adams, Holman Jones et Ellis (2015) appellent a crisis of representation. L'idée que le chercheur puisse être un observateur neutre non impliqué émotionnellement (voir sexuellement) est depuis longtemps enterrée (Kullick, Wilson, 1995, Markowitz, Ashkenazi, 1999), mais c'est d'autant plus impossible lorsqu'il est personnellement concerné par ce qu'il étudie et que sa recherche pénètre sa vie quotidienne. Adams, Holman Jones et Ellis définissent ainsi l'autoethnographie : «a method for exploring, understanding and writing from, through, and with personnal experiences in relation to and in the context of the experiences of others.» (2015: 23). J'ai donc imbriqué l'autoethnographie avec l'amitié comme méthode.

Heureusement j'avançais parallèlement dans mes lectures pour constituer un état de la question et j'ai pu mesurer que l'autoethnographie, même si elle n'était pas nommée en tant que telle, était largement utilisée dans les travaux similaires : B. Soulier (2001), M. Tepper (2015), R. Shuttleworth (2000) y ont eu recours dans leurs thèses sur les blessés médullaires pour les deux premiers et les hommes IMC pour le dernier. S. Smith Rainey (2011) s'appuie elle aussi sur son histoire d'amour avec Max, un homme souffrant de sclérose en plaque, pour construire sa recherche et étayer son analyse. Probablement le recours à l'autoethnographie est-il un peu plus compliqué lorsque l'on travaille sur l'amour. En effet, la charge symbolique de ce sujet rend peutêtre plus suspecte l'utilisation de sa propre expérience comparativement à d'autres champs de la sociologie où cela se fait plus «naturellement » dès qu'il y a observation participante. Mais comme l'expliquent Adams, Holman Jones et Ellis : « Qualitative research (or any research) can no longer maintain a distanced, so-called objective, self-serving stance, and no longer can researchers take advantage of vulnerable others without accounting for their own identities, experiences, social capital, intentions, and formative assumptions » (2015: 18). C'est effectivement l'évolution que l'on observe dans des ouvrages récents sur la Grounded Theory, comme dans The Sage Handbook of Grounded Theory 
(2007) où la place du self du chercheur dans le processus de recherche est plusieurs fois discutée.

L'autoethnographie m'apparaît incontournable lorsqu'on travaille sur un objet qui a une grande part d'intime. C'est le seul moyen d'accéder à l'observation directe de la vie privée : celle d'autres couples et sa propre vie intime, même si la position de la partenaire ordinaire est différente de celle du partenaire médullaire. On peut comprendre la routine, le quotidien, les détails, les anecdotes, les espaces, les moments, les objets, les pratiques, les émotions, y compris ce qui est difficile à dire, ou ce qui n'a pas de nom parce que c'est trop particulier et propre à un groupe. Pour rendre compte de certaines expériences, j'ai dû créer des mots (comme l'«orgasme vicariant»). L'autoethnographie de son propre couple est l'un des seuls moyens d'accéder à la réalité quotidienne et pratique de l'amour, à part quelques places intrusives, comme celle de personal assistant choisie par R. Shuttleworth. Il est regrettable qu'il n'y ait pas plus de chercheurs sur les thèmes de l'amour et/ou de la sexualité qui aient le courage de Ch. Broqua (2000) pour affirmer l'importance de l'implication personnelle explicite et réflexive dans le processus de recherche. On retrouve ce type de réflexions dans les textes réunis par D. Kulick et M. Wilson dans leur ouvrage Taboo. Sex, Identity and Erotic Subjectivity in Anthropological Fieldwork (1995) ou Markowitz F. et Ashkenazi M. dans Sex, Sexuality, and the Anthropologist (1999). Mais elles concernent le plus souvent la sexualité (cf. les titres choisis), qui bien qu'en lien avec l'amour ne se confond pas avec lui, et/ou le genre, un système de catégorisation qui s'efface lorsque rentre en jeu celui du handicap. Dans la plupart des cas les relations se construisent après l'entrée sur un terrain lointain et exotique, où l'altérité est source de désir tout autant qu'elle enrichit le recueil. Il s'agit de relations bien différentes de celles que j’ai pu nouées en tant que femme handicapée avec des hommes handicapés que je connaissais parfois avant de commencer ma recherche. J'étais peut-être une "halfie» (1999) mais notre proximité s'est accrue durant le processus de recherche, jusqu'à ce que nous devenions des semblables humains bien différents des « others » dont parlent les anthropologues de Taboo (1995). K. Poewe a largement discuté l'amour sur le terrain (1992). Faire de l'observation participante c'est vivre la vie quotidienne du groupe étudié, et l'amour en fait partie. On n'arrête pas plus le flot des interactions amoureuses lorsque l'on part travailler qu'on ne peut l'arrêter lorsque l'on fait de la recherche. L'amour qu'elle a expérimenté sur le terrain lui a permis d'améliorer tant ses données que ses résultats en lui permettant de mieux comprendre le groupe qu'elle étudiait.

\section{Conclusion}

J'ai essayé dans cet article de montrer les apports complémentaires de chacun des types de données que l'on peut recueillir sur l'amour. Ces données sont placées sur un continuum entre public/culturel et privé/intime/personnel. D’un côté on peut avoir une très large population qui garantit la variété des cas, de l'autre une population de plus en plus réduite, jusqu'à soi-même, plus subjective, mais qui garantit la profondeur et la durée de l'observation des processus interpersonnels de l'amour. En réunis- 
sant un grand nombre d'histoires qui me sont extérieures, j'ai pu mesurer ce que mon expérience a de partagé, mais c'est parce que j’ai éprouvé personnellement, matériellement et émotionnellement, la perméabilisation des systèmes de catégorisations sociales que j'ai pu comprendre les processus de renégociation des normes et rôles de genre au sein des couples formés par les hommes handicapés. Les données documentaires sont essentielles pour connaître les scripts amoureux proposés aux acteurs par la culture et comprendre l'enjeu des négociations lorsque se confrontent les scripts personnels. Je voudrais conclure sur le potentiel synergique du croisement des méthodes. Il est classique de décrire les savoirs ethnographiques comme des savoirs sensuels, mais le recours à l'échange, l'observation, l'art, la fiction, l'autoethnographie génère en plus des savoirs d'ordre émotionnel. La co-construction d'une relation basée sur les capacités et les aspirations plus que sur les rôles de genres dominants crée, au sein du couple, une gratitude mutuelle qui fait percevoir la relation amoureuse comme exceptionnelle. Mais il m'a fallu l'entendre, le voir, le lire, le vivre pour le ressentir, le savoir et pouvoir le théoriser.

\section{Bibliographie}

Adams T. E., Holman Jones S., Ellis C. (2015), Autoethnography. Understanding Qualitative Research, Oxford, Oxford University Press.

BACHELARD G. (1967), La Formation de l'esprit scientifique, Paris, $5^{e}$ édition, Librairie philosophique J. Vrin.

Barthes R. (1977), Fragments d'un discours amoureux, Paris, Éditions du Seuil.

BECKER H. S. (2002), Les ficelles du métier. Comment conduire sa recherche en Sciences Sociales, Paris, Éditions La Découverte.

BRoQua C. (2000), « Enjeux des méthodes ethnographiques dans l'étude des sexualités entre hommes », Journal des anthropologues, vol. 82-83, p. 129-155.

Bryant A., Charmaz K. (dir.) (2007), The Sage Handbook of Grounded Theory, Los Angeles, Sage.

BouRdieu P. (dir.) (1993), «Comprendre», in La misère du monde, Éditions du Seuil, p. 903-925.

CouRDURIEs J. (2011), 'Être en couple (gay). Conjugalité et homosexualité masculine en France, Lyon, Presses Universitaires de Lyon.

Gagnon J., Simon W. (1973), Sexual Conduct: The Social Sources of Human Sexuality, Chicago, Aldine.

GLASER B. B., StRauss A. L. (2010 [1967]), La découverte de la théorie ancrée. Stratégies pour la recherche qualitative, Paris, Armand Colin. 
GRANJA F. (2013), Vivir el sexo. El hombre que aprendio a vibrar, Barcelone, Luciérnaga Nova.

Holton G. (1981 [1978]), L’imagination scientifique, Paris, Gallimard.

Kосн F. (1994), La vie après l'accident, le bouleversant témoignage des handicapés, Paris, Plon.

Kulick D., Willson M. (dir.) (1995), Taboo: Sex, Identity and Erotic Subjectivity in Anthropological Fieldwork, Londres, Routledge.

Markowitz F., Ashkenazi M. (dir.) (1999), Sex, Sexuality, and the Anthropologist, Chicago, University of Illinois Press.

Moss G. L. (1997 [1996]), L’image de l'homme. L'invention de la virilité moderne, Paris, Abbeville (« Tempo »).

Mouget A. C. (2012), Le chat de la voisine, s.l., TheBookEdition.

Poewe K. (1982), Reflections of a Woman Anthropologist: No Hiding Place, Londres-New York, Academic Press.

Shuttleworth R. (2000), The Pursuit of Sexual Intimacy for Men with Cerebral Palsy, PhD dissertation, San Francisco, University of San Francisco.

Smith Rainey S. (2011), Love, Sex and Disability. The pleasures of Care, Boulder, Rienner.

Shakespeare T., Gillespie-Sells K., Davies D. (1996), The Sexual Politics of Disability: Untold Desires, Londres, Cassell.

SOULIER B. (2001), Aimer au delà du handicap. Vie affective et sexualité du paraplégique, Paris, Dunod.

Tepper M. (2015), Regain That Feeling. Secrets to Sexual Self-Dicovery, s.l., Create Space Selfpublishing.

Tillmann L. M. (dir.) (2003), «Friendship as Method», Qualitative Inquiry, vol. 9, n 5 , p. $729-749$ 